\title{
Acesso da população idosa aos serviços de saúde bucal: uma revisão
} Access of elderly people to oral health services: a review

\author{
Adriana Maria Fuzer Grael Tinós* \\ Silvia Helena de Carvalho Sales-Peres ${ }^{* *}$ \\ Luciane Cristine Ribeiro Rodrigues ${ }^{* * *}$
}

\section{Resumo}

A transição demográfica, no Brasil, implicou em substancial mudança no desenho da pirâmide etária, resultante do aumento da expectativa de vida, devendo os serviços de saúde estar preparados para absorver essa nova demanda. Com relação à saúde bucal da população idosa brasileira, sua condição, assim como em diversos países, é precária. Objetivo: este estudo objetivou conhecer, por meio de revisão bibliográfica, os fatores que interferem no acesso da população idosa aos serviços de saúde bucal. Materiais e método: foi realizada pesquisa nas bases de dados BVS, LILACS, SCIELO e $B B O$, utilizando os descritores: acesso aos serviços de saúde; saúde bucal; idoso. Os critérios de inclusão para seleção dos artigos consistiram na presença do texto completo nas bases de dados, nas publicações que apresentaram como limite de assunto o termo idoso e nos artigos publicados no período compreendido entre os anos de 2001 e 2012. Resultados: o acesso dos idosos aos serviços odontológicos não ocorre de forma regular. Baixo nível de escolaridade, baixa renda, edentulismo, baixa oferta de serviço público odontológico, falta de informação sobre saúde bucal e residir na zona rural podem interferir de forma negativa no acesso dessa população aos serviços de saúde bucal. Considerações finais: limitações relacionadas aos aspectos socioeconômicos constituem-se na principal barreira no acesso de idosos aos serviços odontológicos.

Palavras-chave: Acesso aos serviços de saúde. Assistência odontológica para idosos. Desigualdades em saúde. Idoso. Saúde bucal.

\section{Introdução}

O termo acesso pode ser definido como o ato de ingressar ${ }^{1}$. Segundo Jesus e Assis ${ }^{2}$ (2010), ao estabelecer uma relação dessa definição com os serviços de saúde, o acesso pode ser entendido como a "porta de entrada", o local de acolhimento do usuário no momento de expressão de sua necessidade e, de certa forma, os caminhos percorridos por ele no sistema em busca da resolução dessa necessidade. No Brasil, com a criação do Sistema Único de Saúde (SUS), o acesso à saúde de forma universal, integral e equitativa passou a ser um direito estendido a todos os segmentos da população, cuja estrutura tem apresentado importantes alterações em consequência da transição demográfica, a qual implicou em substancial mudança no desenho da pirâmide etária em razão do aumento da expectativa de vida ${ }^{3}$.

A população idosa acima de 65 anos cresceu $45,9 \%$ entre os anos de 1980 e $2000^{3}$ e, segundo as projeções populacionais com base no Censo Demográfico 2010, recentemente divulgadas pelo IBGE, esse crescimento tende a continuar, uma vez que, em 2060, o percentual dessa população será de $26,8 \%$, enquanto em 2013 é de 7,4\% .

A transição demográfica vem acompanhada da transição epidemiológica, caracterizada pela mudança do perfil de morbidade e de mortalidade de

** Professora doutora associada do Departamento de Odontopediatria, Ortodontia e Saúde Coletiva, Faculdade de Odontologia de Bauru, USP, Bauru, SP, Brasil. Orientadora de TCC da Unifesp São Paulo, Enfermeira, Doutoranda do Departamento de Saúde Coletiva da Universidade Estadual Paulista (Unesp), Botucatu, SP, Brasil. 
uma população, com diminuição progressiva das mortes por doenças infectocontagiosas e elevação das mortes por doenças crônicas ${ }^{3}$. No Brasil, particularmente, essa transição ocorre de forma atípica, decorrente não só da reemergência e "permanência" de doenças infecciosas e parasitárias como também das chamadas causas externas, expressão da violência social em suas mais diversas formas, modelo que é conhecido como "mosaico epidemiológico"5.

Enquanto nos países desenvolvidos a transição epidemiológica transcorreu em um período longo, nos países em desenvolvimento ocorre de maneira rápida, acarretando profundas necessidades de adaptação dos serviços de saúde às novas realida$\operatorname{des}^{3}$. Porém, até o final de 2002, o idoso brasileiro não contava, e ao longo da vida não contou, com um sistema de atenção voltado para a promoção de um envelhecimento bem-sucedido. Diante dessa realidade, fazia-se necessário reforçar os princípios do SUS - universalidade, integralidade, equidade - e destacar sua importância, já que, na época, $73 \%$ dos idosos brasileiros dependiam exclusivamente do sistema público de saúde ${ }^{6}$.

Com relação à saúde bucal da população idosa brasileira, sua condição, como em diversos países, é precária $^{7,8}$, possivelmente em decorrência do caráter excludente da atenção odontológica pública vigente no passado ${ }^{9}$. De acordo com os resultados do último levantamento das condições de saúde bucal do brasileiro, realizado no ano de 2010 pelo Ministério da Saúde - SB Brasil 2010 -, o CPOD médio na faixa etária de 65 a 74 anos foi de 27,53, sendo o componente perdido responsável por cerca de $90 \%$ do índice. O mesmo levantamento constatou, também nessa faixa etária, que 14,7\% nunca consultaram um dentista ao longo da vida, que a prevalência de necessidade de tratamento dentário era de $46,6 \%$ e que apenas $7,3 \%$ não necessitavam de prótese dentária ${ }^{10}$.

Diante da atual situação epidemiológica dos idosos brasileiros e do fato de a proporção dessa população estar aumentando em ritmo bastante acelerado, seria importante conhecer o padrão do acesso dessa parcela populacional aos serviços de saúde bucal, o que possibilitaria não somente verificar a qualidade desse acesso como também disponibilizar aos gestores dados para a realização de planejamento estratégico da oferta desses serviços, corroborando para melhorias na qualidade de vida dos idosos brasileiros. Dessa forma, o objetivo deste estudo foi conhecer, por meio de uma revisão bibliográfica, os fatores que interferem no acesso da população idosa aos serviços de saúde bucal.

\section{Materiais e método}

O presente estudo, do tipo revisão bibliográfica, utilizou como método a busca ativa de informações, sob a forma de artigos científicos selecionados me- diante consulta às bases de dados BVS, LILACS, SciELO e BBO, realizada no mês de agosto de 2012 . Considerando o tema central deste trabalho - acesso da população idosa aos serviços de saúde bucal -, empregou-se, na pesquisa, os seguintes descritores, no idioma português, obtidos junto ao DeCS/MeSH: acesso aos serviços de saúde; saúde bucal; idoso.

Para a seleção dos artigos, foram estabelecidos alguns critérios de inclusão, aplicados nesta sequência: i) presença do texto completo nas bases de dados; ii) publicações que apresentaram como limite de assunto o termo idoso; iii) artigos publicados entre os anos de 2001 e 2012. Tal período foi selecionado pelo fato de poder abranger publicações relevantes sobre o tema, uma vez que foram realizados, pelo Ministério da Saúde, dois importantes levantamentos das condições de saúde bucal - SB 2000 e SB Brasil 2010 -, os quais revelaram a preocupante realidade epidemiológica dessa população. Além disso, a partir de 2001, passaram a ser implantadas as Equipes de Saúde Bucal no Programa Saúde da Família (PSF), com a proposta de reorganização do modelo de atenção em saúde bucal. Acrescente-se, ainda, nesse período, o surgimento da Lei no 10.741 , de $1^{\circ}$ de outubro de 2003, que dispôs sobre o Estatuto do Idoso; o lançamento, também em 2003, da Política Nacional de Saúde Bucal - Programa Brasil Sorridente, com vistas à reorganização da prática e à qualificação das ações e dos serviços de saúde bucal; bem como a publicação da Portaria n ${ }^{0} 2.528$, de 19 de outubro de 2006, que aprovou a Política Nacional de Saúde da Pessoa Idosa.

O processo de seleção dos artigos ocorreu no período entre agosto e outubro de 2012. Durante a busca inicial, que contou com a participação de um dos autores (AMFGT), foram selecionados os artigos que obedeceram aos critérios acima descritos. A seguir, foi realizada por todos os autores uma leitura exploratória dos textos, iniciando-se pelos títulos e resumos, a fim de confirmar se os materiais selecionados tratavam realmente do objeto a ser explorado. Nos casos em que o objetivo da pesquisa não era apresentado de forma clara no resumo, houve a necessidade da leitura do artigo completo. Nessa etapa, excluiu-se os que se apresentavam em duplicata, assim como aqueles cujo link "texto completo", informado nas bases de dados, se encontrava indisponível. Foram desconsideradas, ainda, as publicações em forma de editorial, resumo de anais e relato de experiência, em razão das limitações dos dados. Em seguida, procedeu-se à leitura seletiva dos artigos no intuito de eleger apenas aqueles que abordavam, especificamente, a proposição deste estudo. Dada a escassez de artigos envolvendo exclusivamente a população idosa, convencionou-se não descartar aqueles que abrangiam, além dos idosos, outras faixas etárias. Ressalta-se que divergências, por parte dos autores, com relação à elegibilidade dos artigos foram resolvidas por consenso. 
Definidos os trabalhos que seriam utilizados na realização da pesquisa, estes foram impressos, lidos na íntegra e analisados segundo as variáveis que seguem: ano, país e revista de publicação; objetivo do estudo; metodologia utilizada - delineamento/tipo de estudo, tipo de fonte de dados (primária/secundária), fonte dos dados e forma de coleta, faixa etária da população de estudo -; e resultados/conclusões.

\section{Resultados}

A utilização dos descritores, acima mencionados, nas bases de dados permitiu a localização de 321 artigos, sendo 301 na BVS, 14 na LILACS, 5 na BBO e 1 na biblioteca virtual SciELO. Após a aplicação dos critérios de inclusão e exclusão referidos anteriormente, foram selecionados 11 trabalhos na literatura científica para a realização deste estudo. A Figura 1 mostra o processo de seleção dos artigos a partir de sua localização nas bases de dados.

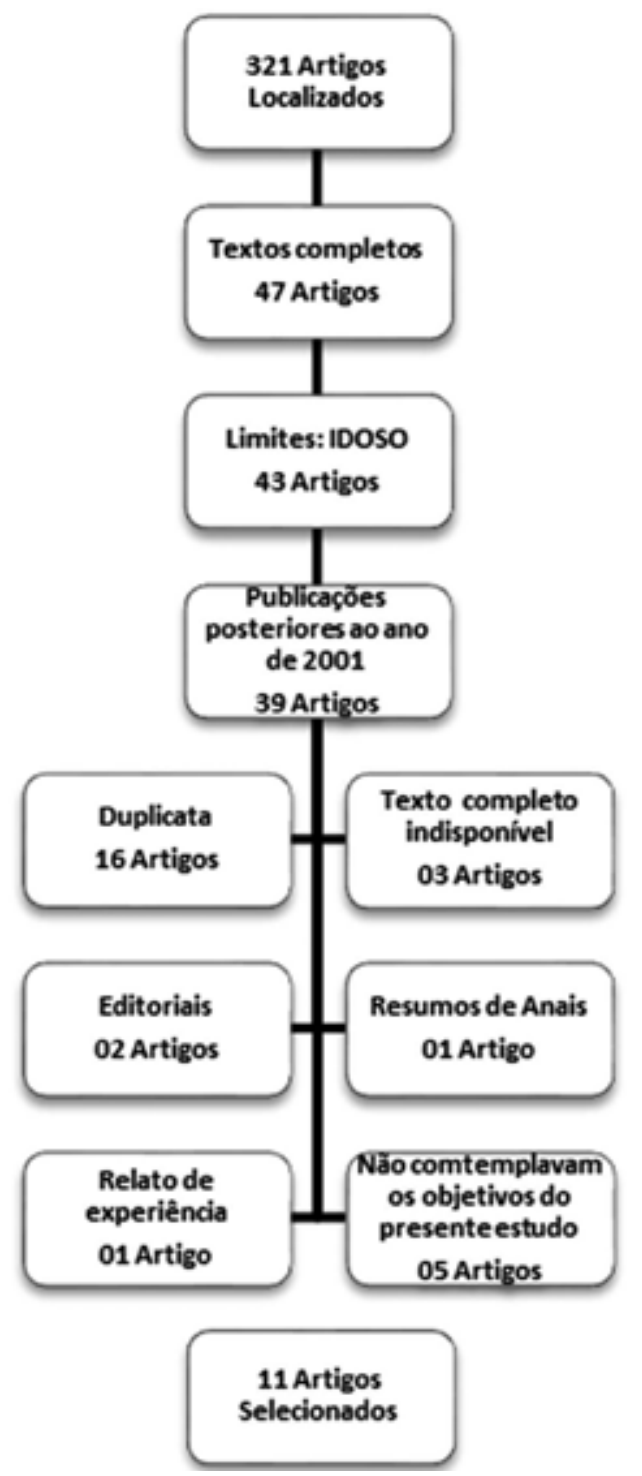

Figura 1 - Processo de seleção dos artigos considerando os critérios de inclusão pré-estabelecidos e as exclusões realizadas durante o processo
A seleção permitiu identificar estudos publicados a partir do ano de 2005, sendo quatro ${ }^{11-14}$ de 2008 e dois $^{15,16}$ de 2010 . Os demais estudos ${ }^{17-21}$ apresentaram distribuição uniforme, com uma publicação/ano. Quanto ao país de realização do estudo, nove eram do Brasil ${ }^{11-17,19,21}$, um da Suécia $^{20}$ e um da Nova Zelândia ${ }^{18}$. Maior frequência de publicações $(\mathrm{n}=4)^{11-13,19}$ foi observada no periódico Cadernos de Saúde Pública.

Dos onze artigos, seis avaliaram o acesso ${ }^{11,13,14,16,19,20}$, dois a utilização ${ }^{15,21}$ e um o uso de serviços odontológicos ${ }^{12}$. Apenas dois ${ }^{17,18}$ não objetivaram estudar, de forma direta, os temas anteriores.

Foi possível identificar alguns fatores que podem interferir de modo negativo no acesso aos serviços de saúde bucal: baixo nível de escolaridade $11-15,17,19-21$; baixa renda ${ }^{11-13,19,20}$; edentulismo ${ }^{15,21}$; baixa oferta de serviço público odontológico ${ }^{19}$; falta de informação sobre saúde bucal ${ }^{21}$ e residir na zona rural ${ }^{12}$. A Tabela 1 mostra a distribuição dos artigos segundo 0 ano, país em que foi realizado o estudo, revista de publicação, objetivo e principais resultados. 


\begin{tabular}{|c|c|c|c|c|c|}
\hline Autores & Ano & País & Revista & Objetivo do estudo & Principais resultados \\
\hline Moreira et al. ${ }^{19}$ & 2005 & Brasil & $\begin{array}{l}\text { Cad Saúde } \\
\text { Pública }\end{array}$ & $\begin{array}{l}\text { Conhecer o quadro } \\
\text { epidemiológico da saúde bucal } \\
\text { dos idosos brasileiros e seu } \\
\text { acesso aos serviços de atenção } \\
\text { odontológica. }\end{array}$ & $\begin{array}{l}\text { - Principais barreiras para o acesso: baixa es- } \\
\text { colaridade, baixa renda e escassa oferta de } \\
\text { serviços públicos odontológicos voltados à } \\
\text { população idosa brasileira. }\end{array}$ \\
\hline $\begin{array}{l}\text { Wamala, Merlo } \\
\text { e Boström }{ }^{20}\end{array}$ & 2006 & Suécia & $\begin{array}{l}\text { J Epidemiol } \\
\text { Community } \\
\text { Health }\end{array}$ & $\begin{array}{l}\text { Analisar os efeitos da desvantagem } \\
\text { socioeconômica no acesso aos } \\
\text { serviços odontológicos em saúde } \\
\text { bucal. }\end{array}$ & $\begin{array}{l}\text { - Pouca idade, baixos níveis de escolaridade } \\
\text { e ocupação e viver sozinho foram associados } \\
\text { à não procura por tratamento odontológico, } \\
\text { sendo as limitações financeiras a principal ra- } \\
\text { zão para a ocorrência de tal desfecho. }\end{array}$ \\
\hline $\begin{array}{l}\text { Martins, Barreto } \\
\text { e Pordeus }{ }^{21}\end{array}$ & 2007 & Brasil & $\begin{array}{l}\text { Rev Panam } \\
\text { Salud Publica }\end{array}$ & $\begin{array}{l}\text { Investigar a utilização de } \\
\text { serviços odontológicos e seus } \\
\text { determinantes entre idosos } \\
\text { brasileiros. }\end{array}$ & $\begin{array}{l}\text { - A menor prevalência de uso de serviços } \\
\text { odontológicos no último ano foi entre os ido- } \\
\text { sos com mais baixa escolaridade, que relata- } \\
\text { ram não ter recebido informações sobre saúde } \\
\text { bucal, edentados e que mais necessitavam de } \\
\text { tratamento, sugerindo iniquidade no acesso } \\
\text { aos serviços odontológicos. }\end{array}$ \\
\hline $\begin{array}{l}\text { Manhães e } \\
\text { Costa }^{11}\end{array}$ & 2008 & Brasil & $\begin{array}{l}\text { Cad Saúde } \\
\text { Pública }\end{array}$ & $\begin{array}{l}\text { Analisar o acesso a e a utilização } \\
\text { de serviços odontológicos no } \\
\text { estado do Rio de Janeiro em 1998, } \\
\text { a partir da Pesquisa Nacional por } \\
\text { Amostra de Domicílios (PNAD, } \\
\text { 1998). }\end{array}$ & $\begin{array}{l}\text { - 2,6\% da população de idosos relataram nun- } \\
\text { ca ter ido ao dentista na vida. } \\
\text { - Queda do padrão socioeconômico (renda per } \\
\text { capita, escolaridade, classe econômica e pla- } \\
\text { no de saúde) corroborou para o aumento da } \\
\text { privação do acesso aos serviços odontológicos. }\end{array}$ \\
\hline $\begin{array}{l}\text { Martins, Barreto } \\
\text { e Pordeus }{ }^{12}\end{array}$ & 2008 & Brasil & $\begin{array}{l}\text { Cad Saúde } \\
\text { Pública }\end{array}$ & \begin{tabular}{|l|} 
Investigar o uso de serviços \\
odontológicos entre idosos \\
dentados e edentados residentes \\
na Região Sudeste do Brasil, \\
participantes do inquérito de saúde \\
bucal realizado pelo Ministério da \\
Saúde em 2002/2003.
\end{tabular} & $\begin{array}{l}\text { - Constatou-se iniquidade no acesso aos servi- } \\
\text { ços odontológicos e no seu uso. } \\
\text { - Entre os dentados, os residentes na zona rural, } \\
\text { com menor renda e que necessitavam de pró- } \\
\text { tese usaram menos os serviços odontológicos; } \\
\text { entre os edentados, aqueles com menor escola- } \\
\text { ridade foram menos ao dentista. }\end{array}$ \\
\hline Rocha e Góes ${ }^{13}$ & 2008 & Brasil & $\begin{array}{l}\text { Cad Saúde } \\
\text { Pública }\end{array}$ & \begin{tabular}{|l|} 
Avaliar fatores associados ao \\
acesso aos serviços de saúde bucal \\
comparando as áreas cobertas e \\
não cobertas pela Estratégia de \\
Saúde da Família (ESF).
\end{tabular} & $\begin{array}{l}\text { - Não existiu diferença no acesso aos serviços } \\
\text { de saúde bucal entre os residentes em áreas } \\
\text { cobertas e não cobertas pela ESF. } \\
\text { - } 67,5 \% \text { dos idosos não tiveram acesso aos ser- } \\
\text { viços públicos de saúde bucal. }\end{array}$ \\
\hline $\begin{array}{l}\text { Costa, Maciel e } \\
\text { Cavalcanti }{ }^{14}\end{array}$ & 2008 & Brasil & $\begin{array}{l}\text { Odontologia } \\
\text { Clín-Científ }\end{array}$ & $\begin{array}{l}\text { Avaliar o acesso aos serviços } \\
\text { odontológicos e os motivos da } \\
\text { procura por atendimento por } \\
\text { idosos de Campina Grande/PB. }\end{array}$ & $\begin{array}{l}\text { - Constatou-se baixo nível educacional da po- } \\
\text { pulação de estudo, com a maioria dos idosos } \\
(62,9 \%) \text { apresentando o ensino fundamental } \\
\text { incompleto. } \\
-69,4 \% \text { dos idosos relataram um período de } \\
\text { mais de três anos da última visita ao dentista e } \\
71 \% \text { referiram necessidade de tratamento. }\end{array}$ \\
\hline Baldani et al. ${ }^{15}$ & 2010 & Brasil & $\begin{array}{l}\text { Rev Bras } \\
\text { Epidemiol }\end{array}$ & \begin{tabular}{|l|} 
Identificar os fatores individuais \\
associados à utilização de serviços \\
odontológicos por parte de adultos \\
e idosos de baixa renda residentes \\
na área de abrangência da ESF.
\end{tabular} & $\begin{array}{l}\text { - } 67 \% \text { dos idosos não iam ao dentista há três } \\
\text { anos ou mais, e } 38 \% \text { acreditavam necessitar } \\
\text { de tratamento odontológico. }\end{array}$ \\
\hline Alcarde et al. ${ }^{16}$ & 2010 & Brasil & $\begin{array}{l}\text { Rev Odonto } \\
\text { Ciênc }\end{array}$ & \begin{tabular}{|l|} 
Medir a autopercepção das \\
condições de saúde bucal de 137 \\
idosos, considerando a influência \\
de variáveis sociodemográficas, \\
situação de institucionalização e \\
acesso aos serviços de saúde bucal \\
sobre a pontuação final do índice \\
GOHAI.
\end{tabular} & $\begin{array}{l}\text { - Apenas } 32 \% \text { dos idosos tinham consultado } \\
\text { um dentista no último ano, sendo } 28 \% \text { no ser- } \\
\text { viço público de saúde. } \\
\text { - A média de pontuação GOHAl foi de } 27,5 \text {, } \\
\text { indicando uma baixa autopercepção da saúde } \\
\text { bucal da amostra, sendo menor entre os idosos } \\
\text { institucionalizados e que vivem sozinhos ou } \\
\text { fora do ambiente familiar. }\end{array}$ \\
\hline Alcântara et al. ${ }^{17}$ & 2011 & Brasil & $\begin{array}{l}\text { PHYSIS: Rev } \\
\text { Saúde Coletiva }\end{array}$ & \begin{tabular}{|l|} 
Comparar a condição de \\
saúde bucal de idosos não \\
institucionalizados com a meta da \\
OMS para o ano de 2010.
\end{tabular} & $\begin{array}{l}\text { - } 39,1 \% \text { relataram ter ido ao dentista no últi- } \\
\text { mo ano, e, destes, } 41 \% \text { utilizaram o serviço } \\
\text { público. } \\
\text { - O edentulismo permanece como importan- } \\
\text { te problema de saúde bucal entre os idosos } \\
\text { examinados, indicando que as metas da OMS } \\
\text { para o ano de } 2010 \text { não foram cumpridas. }\end{array}$ \\
\hline Gregory et al. ${ }^{18}$ & 2012 & $\begin{array}{c}\text { Nova } \\
\text { Zelândia }\end{array}$ & Gerodontology & $\begin{array}{l}\text { Explorar experiências e percepções } \\
\text { de saúde bucal e de cuidados } \\
\text { de saúde oral entre uma amostra } \\
\text { etnicamente mista de idosos } \\
\text { neozelandeses. }\end{array}$ & $\begin{array}{l}\text { - Foram identificados alguns fatores que po- } \\
\text { dem interferir no acesso aos serviços de saúde } \\
\text { bucal: recursos financeiros; transporte/mobili- } \\
\text { dade; disponibilidade de tempo; autopercep- } \\
\text { ção da necessidade de tratamento. }\end{array}$ \\
\hline
\end{tabular}


Com relação à metodologia utilizada pelos autores, do total de estudos analisados, sete eram artigos de análise de dados secundários ${ }^{11,12,15,17,19-21}$ (dois utilizaram o SB2000) e quatro utilizaram dados primários, sendo três quantitativos ${ }^{13,14,16}$ - do tipo trans- versal - e um qualitativo ${ }^{18}$. Quanto à faixa etária da população de estudo, sete artigos abordaram especificamente a população idosa ${ }^{12,14,16,17-19,21}$. Os resultados referentes à metodologia utilizada nos estudos selecionados encontram-se dispostos na Tabela 2 .

Tabela 2 - Características dos estudos de acordo com o método utilizado

\begin{tabular}{|c|c|c|c|c|}
\hline $\begin{array}{l}\text { Estudos } \\
\text { selecionados }\end{array}$ & $\begin{array}{l}\text { Delineamento/ } \\
\text { Tipo de } \\
\text { estudo }\end{array}$ & $\begin{array}{l}\text { Tipo de } \\
\text { fonte de } \\
\text { dados }\end{array}$ & Fonte dos dados e forma de coleta & $\begin{array}{c}\text { Faixa etária da } \\
\text { população de estudo }\end{array}$ \\
\hline $\begin{array}{l}\text { Moreira et } \\
\text { al. }{ }^{19}\end{array}$ & $\begin{array}{l}\text { Revisão } \\
\text { sistemática }\end{array}$ & Secundários & Bases de dados & - \\
\hline $\begin{array}{l}\text { Wamala, } \\
\text { Merlo e } \\
\text { Boström }{ }^{20}\end{array}$ & $\begin{array}{l}\text { Artigo de } \\
\text { análise }^{*}\end{array}$ & Secundários & $\begin{array}{l}n=37.399 / \text { Swedish National Surveys of Public } \\
\text { Health } 2004 \text { and } 2005\end{array}$ & $\begin{array}{l}21 \text { a } 35 ; 35 \text { a } 50 ; 50 \\
\text { a } 65 \text { e } 65 \text { a } 84 .\end{array}$ \\
\hline $\begin{array}{l}\text { Martins, } \\
\text { Barreto e } \\
\text { Pordeus }^{21}\end{array}$ & $\begin{array}{l}\text { Artigo de } \\
\text { análise }^{*}\end{array}$ & Secundários & SB 2000 & 65 a 74 anos \\
\hline $\begin{array}{l}\text { Manhães e } \\
\text { Costa }^{11}\end{array}$ & $\begin{array}{l}\text { Artigo de } \\
\text { análise }\end{array}$ & Secundários & PNAD 1998 & $\begin{array}{l}15 \text { a } 19,35 \text { a } 44 \text { e } 65 \\
\text { a } 74 \text { anos }\end{array}$ \\
\hline $\begin{array}{l}\text { Martins, } \\
\text { Barreto e } \\
\text { Pordeus }^{12}\end{array}$ & $\begin{array}{l}\text { Artigo de } \\
\text { análise* }\end{array}$ & Secundários & SB 2000 & 65 a 74 anos \\
\hline $\begin{array}{l}\text { Rocha e } \\
\text { Góes }^{13}\end{array}$ & Transversal & Primários & $\begin{array}{l}\mathrm{n}=827 / \text { Observação direta intensiva por meio } \\
\text { de formulário }\end{array}$ & $\begin{array}{l}18 \text { a } 24,25 \text { a } 49,50 \\
\text { a } 64 \text { e } 65 \text { ou }+ \text { anos }\end{array}$ \\
\hline $\begin{array}{l}\text { Costa, Maciel } \\
\text { e Cavalcanti }\end{array}$ & Transversal & Primários & $\begin{array}{l}\mathrm{n}=124 / \text { Aplicação de questionário a } \\
\text { participantes de grupos de convivência }\end{array}$ & $\geq 60$ anos \\
\hline $\begin{array}{l}\text { Baldani, et } \\
\text { al. }^{15}\end{array}$ & $\begin{array}{l}\text { Artigo de } \\
\text { análise* }\end{array}$ & Secundários & $\begin{array}{l}\mathrm{n}=246 / \text { Estudo de base populacional que } \\
\text { teve por objetivo identificar desigualdades na } \\
\text { utilização e no acesso a tratamento odontológico } \\
\text { na área de abrangência da Estratégia Saúde da } \\
\text { Família em Ponta Grossa-PR. }\end{array}$ & $\begin{array}{l}35 \text { a } 59 \text { e } 60 \text { ou + } \\
\text { anos }\end{array}$ \\
\hline $\begin{array}{l}\text { Alcarde et } \\
\text { al. }^{16}\end{array}$ & Transversal & Primários & $\begin{array}{l}\mathrm{n}=137 / \text { Exame oral e dois questionários: um } \\
\text { primeiro composto pelos } 12 \text { itens do índice } \\
\text { GOHAI, e um segundo para análise das } \\
\text { variáveis sociodemográficas. }\end{array}$ & 60 a 92 anos \\
\hline $\begin{array}{l}\text { Alcântara et } \\
\text { al. }{ }^{17}\end{array}$ & $\begin{array}{l}\text { Artigo de } \\
\text { análise }^{*}\end{array}$ & Secundários & $\begin{array}{l}\mathrm{n}=428 / \text { Levantamento epidemiológico da } \\
\text { saúde bucal de idosos institucionalizados e não } \\
\text { institucionalizados de Governador Valadares- } \\
\text { MG, 2007-2008. }\end{array}$ & $\geq 60$ anos \\
\hline $\begin{array}{l}\text { Gregory et } \\
\text { al. }^{18}\end{array}$ & Qualitativo & Primários & $\begin{array}{l}\mathrm{n}=24 / \text { Entrevista semiestruturada envolvendo } \\
\text { três temas principais: negociação de custo } \\
\text { e conveniência do acesso; limitações na } \\
\text { manutenção da saúde bucal; confiança nos } \\
\text { profissionais de saúde bucal. }\end{array}$ & 60 a 93 anos \\
\hline
\end{tabular}

* Artigos que utilizaram dados secundários já publicados em suas análises

\section{Discussão}

A população brasileira envelhece de forma rápida e intensa $\mathrm{a}^{22}$, e, atualmente, as políticas públicas de saúde, com o objetivo de garantir atenção a todos os cidadãos, têm dado visibilidade à parcela idosa que a compõe. É possível que essa nova postura do sistema tenha contribuído para o aumento do interesse da comunidade científica na produção de estudos que envolvam essa população. 
Tal suposição converge com os resultados encontrados neste estudo quanto ao ano de publicação dos artigos selecionados, uma vez que a quase totalidade destes foi publicada a partir de 2006, com predomínio do ano de 2008. Ressalta-se que em 2006 foi divulgado o Pacto pela Saúde, que surgiu como uma estratégia para viabilizar a equidade em saúde no Brasil ${ }^{23}$. Em uma de suas dimensões - o Pacto pela Vida -, foram elencadas prioridades que apresentam impacto sobre a situação de saúde da população brasileira, sendo uma delas a Saúde do Idoso, cujo objetivo, ainda em 2006, era implantar a Política Nacional de Saúde da Pessoa Idosa, buscando a atenção integral a essa parcela da população ${ }^{24}$. Dentre as ações estratégicas que envolvem a saúde da pessoa idosa, preconizada no referido Pacto, destaca-se a reorganização do processo de acolhimento nas Unidades de Saúde, como forma de enfrentamento das dificuldades atuais de acesso.

Três artigos mencionaram a existência de iniquidade no acesso da população idosa aos serviços de saúde bucal ${ }^{12,20,21}$. Costa et al. ${ }^{14}$ (2008) observaram que $97,6 \%$ dos pesquisados já tinham ido alguma vez ao cirurgião-dentista, porém, destes, $69,4 \%$ referiram um período de mais de três anos desde a última visita. Neste estudo, a maioria correspondeu a usuários do serviço público $(56,5 \%)$, divergindo dos resultados encontrados por Alcarde et al. ${ }^{16}$ (2010) e Alcântara et al. ${ }^{17}$ (2011), em que $41 \%$ e $28 \%$ da população de estudo utilizaram os serviços públicos, respectivamente.

De acordo com o trabalho de Rocha e Góes ${ }^{13}$ (2008), 67,5\% dos idosos não tiveram acesso aos serviços públicos de saúde bucal nos últimos dois anos, não existindo diferença no acesso entre os residentes em áreas cobertas e não cobertas pela ESF. Os mesmos autores relataram, ainda, que, com 0 avançar da idade, ocorre uma redução do acesso aos serviços de saúde bucal, diferentemente do encontrado no estudo sueco desenvolvido por Wamala et al. ${ }^{20}$ (2006), no qual a pouca idade foi associada à não procura por tratamento odontológico.

Já o estudo qualitativo de Gregory et al. ${ }^{18}$ (2012), realizado na Nova Zelândia, identificou dificuldades conflitantes no acesso de idosos aos serviços de saúde bucal, onde a superação de um obstáculo poderia trazer outras limitações. Participantes deste estudo relataram que atendimento odontológico mais barato, necessário para os idosos com baixos rendimentos, poderia ser acessado através da Faculdade de Odontologia, porém o fato de esse serviço exigir um maior número de deslocamentos, em decorrência de tratamentos prolongados, com elevado número de sessões, tornava essa alternativa frustrantemente difícil, considerando, nesse caso, os idosos que dependem de amigos ou parentes para transportá-los.

Moreira et al. ${ }^{19}$ (2005) definiram, em seu trabalho, quatro categorias onde foram agrupados aspectos que podem influenciar o acesso e a utilização dos serviços de saúde pela população idosa brasileira: aspectos sociodemográficos; aspectos indicadores de saúde geral em idosos; aspectos comportamentais/culturais; organização e planejamento das ações de saúde. Optou-se, por questões didáticas, por proceder à discussão dos resultados encontrados neste estudo separando-os de acordo com as categorias propostas pelos autores. Tais resultados são passíveis enquadramento em três das quatro categorias supracitadas:

\section{Aspectos sociodemográficos}

De acordo com Ayres et al. ${ }^{25}$ (2006), a vulnerabilidade social é caracterizada por aspectos culturais, sociais e econômicos que determinam as oportunidades de acesso a bens e serviços. Considerando essa afirmativa, é possível assegurar que a população idosa encontra-se em situação de grande vulnerabilidade social. Segundo dados do IBGE, a proporção de idosos sem instrução e com menos de um ano de estudo, em 2009, era de 30,7\% no conjunto do país ${ }^{26}$.

A baixa escolaridade aparece como um dos principais fatores limitadores do acesso de idosos aos serviços odontológicos, sendo tal aspecto fácil de ser entendido, ao considerar-se que, à medida que o indivíduo acumula anos de estudo, adquire maior conhecimento sobre saúde bucal e passa a entender a importância do uso dos serviços de saúde de forma preventiva ${ }^{27}$. Essa afirmação pode estar relacionada aos resultados encontrados por Martins et al. ${ }^{21}$ (2007), em que a menor prevalência de uso de serviços odontológicos foi observada entre os idosos que, além de apresentarem baixa escolaridade, relataram não ter recebido informações sobre saúde bucal. Os mesmos autores consideram esse último achado como indicativo da necessidade de melhorar, entre essa população, a qualidade dos serviços preventivos - oferecendo orientações sobre o autoexame bucal para a identificação precoce dos problemas bucais - e educativos, com a difusão de informações relacionadas à saúde bucal.

Outro aspecto relacionado que pode influenciar, de forma negativa, o acesso aos serviços de saúde é a baixa renda. Segundo Viana et al. ${ }^{28}$ (2010), populações idosas economicamente desfavorecidas, cuja renda muitas vezes está comprometida com outros membros da família, podem estar limitadas em utilizar serviços odontológicos. É importante salientar que a escolaridade e a renda são aspectos que podem estar profundamente imbricados ${ }^{19}$. Neri e Soares $^{29}$ (2002) também observaram em seu estudo que o aumento das chances de procurar serviços de saúde estaria diretamente relacionado a grupos sociais mais privilegiados, ou seja, de maior escolaridade e com acesso aos serviços públicos (água, luz, esgoto, coleta de lixo) e a plano de saúde.

A Pesquisa Nacional por Amostra de Domicílios (PNAD), realizada em 2008, revelou que o percentual de pessoas com 65 anos ou mais de idade cober- 
tas por plano de saúde, seja de empresas privadas ou de assistência ao servidor público, era de $29,7 \%$. Porém tal cobertura estava positivamente relacionada ao rendimento mensal domiciliar per capita ${ }^{30}$. No Brasil, uma das características marcantes da população que envelhece é a pobreza ${ }^{31}$. Em 2009, $32 \%$ dos idosos possuíam rendimento mensal domiciliar per capita na classe de $1 / 2$ a 1 salário mínimo, enquanto em $11,2 \%$ esse rendimento se apresentava inferior a $1 \frac{1}{2}$ salário mínimo, caracterizando situação de pobreza ${ }^{26}$.

De acordo com Rodrigues e $\mathrm{Neri}^{32}$ (2012), quanto mais velho e mais pobre o indivíduo, menor a frequência de visitas ao dentista e pior a qualidade da sua saúde bucal, que também se nutre dos déficits em cuidados acumulados ao longo da vida. Dessa forma, diante da atual realidade, no que se refere ao perfil socioeconômico da população idosa e à baixa oferta de serviços públicos odontológicos, faz-se necessário uma reformulação do setor com vistas a ampliar o acesso desse segmento a esses serviços.

\section{Aspectos comportamentais/culturais}

Menor prevalência de uso dos serviços odontológicos também foi observada entre os idosos edentados, o que pode estar relacionado ao fato de estes acreditarem que visitar o dentista regularmente é importante apenas para quem tem dentes.

O modelo excludente e o padrão mutilador da prática odontológica dirigida a esse grupo no passado tornaram o edentulismo uma condição frequente no Brasil. Ao considerar a importância de consultar um cirurgião-dentista, tanto para avaliar a necessidade de utilização ou substituição de próteses dentárias como para diagnosticar precocemente lesões potencialmente cancerígenas ${ }^{33}$, fica evidente a necessidade de conhecer as crenças sobre a utilização dos serviços odontológicos nessa população específica ${ }^{12,21}$. Viana et al. ${ }^{28}$ (2010) indicam a questão dos "valores" como possível ponto de entrave para o desejo de cuidados odontológicos, porque, culturalmente, nesse momento da vida, a atenção à saúde muitas vezes está dirigida aos serviços médicos. Dados da PNAD $2008^{30}$ reforçam essa afirmação, pois apontam que cerca de $80 \%$ da população de 65 ou mais anos consultou um médico nos últimos 12 meses, enquanto algo em torno de $64 \%$ desse mesmo segmento consultou um dentista pela última vez há três anos ou mais.

Cabe destacar que o edentulismo constitui-se num quadro irreversível entre os atingidos, com impacto direto na qualidade de vida. Segundo Colussi e Freitas ${ }^{7}$ (2002), além de medidas educativas e preventivas, deve-se pensar em medidas reabilitadoras, nesses casos especificamente. Os mesmos autores defendem, ainda, que a implementação de um serviço de prótese dentária no setor público é uma medida viável e que deveria ser encarada como profilática, uma vez que a falta de dentes acarreta outros problemas de saúde, agravando os já existentes e piorando a qualidade de vida da população idosa brasileira.

\section{Organização e planejamento das ações de saúde}

A escassa oferta de serviços públicos de atenção à saúde bucal também foi relatada por Moreira et al. ${ }^{19}$ (2005) como uma das principais barreiras no acesso aos serviços odontológicos. Levando em conta o perfil socioeconômico dos idosos brasileiros, percebe-se que boa parte dessa população tende a depender diretamente dos serviços públicos de saúde. Porém, resultado preocupante da Pesquisa Nacional por Amostra de Domicílios (PNAD 2008), em seu Suplemento de Saúde ${ }^{30}$, mostra que um número expressivo de idosos $(32,5 \%)$ não tinha seu domicílio cadastrado no PSF nem tinha plano de saúde particular. Os resultados encontrados por Alcarde et al. ${ }^{16}(2010)$ e Alcântara et al. ${ }^{17}$ (2011) confirmam esse aspecto, já que menos da metade dos idosos participantes desses estudos $(41 \%$ e $28 \%$, respectivamente) utilizou os serviços públicos no último ano ao da realização da pesquisa.

Há que se considerar, ainda, a qualidade do acolhimento dessa população nas Unidades de Saúde, a qual pode interferir na busca pelo atendimento. Ressalta-se que o acolhimento constitui-se num dos dispositivos de operacionalização da Política Nacional de Humanização $(\mathrm{PNH})^{34}$, daí seu caráter solidário. De um modo geral, acolher bem implica a tolerância à diferença, a inclusão social e a escuta qualificada, responsável e comprometida ${ }^{35}$.

Segundo Santos et al. ${ }^{35}$ (2002), a falta de compromisso dos trabalhadores de saúde com o sofrimento dos usuários, a baixa capacidade resolutiva das ações de saúde, a intensa desigualdade no atendimento dos diferentes estratos econômico-sociais e a vantagem dos cidadãos que podem pagar altos preços pelos serviços, no acesso ao melhor que se tem no setor, demonstram a desumanização do serviço na área da saúde. Com relação ao idoso, atitudes como o estabelecimento de uma relação respeitosa, acreditar que o idoso é capaz de compreender as perguntas que lhe são feitas ou as orientações que lhe são fornecidas, chamá-lo por seu nome e manter contato visual, bem como utilizar uma linguagem clara $^{34}$, podem fazer a diferença no atendimento, favorecendo o acesso e até mesmo uma melhor adesão ao tratamento proposto.

Em adição, para a efetivação do acolhimento da pessoa idosa, os profissionais de saúde devem compreender as especificidades dessa população e a própria legislação brasileira vigente ${ }^{34}$. O Estatuto do Idoso ${ }^{36}$, em seu Capítulo IV, Art. 18, prevê que as instituições de saúde devem atender aos critérios mínimos para o atendimento às necessidades do idoso, promovendo o treinamento e a capacitação dos profissionais. Logo, é importante que os profissionais de saúde que lidam com pacientes idosos co- 
nheçam o conjunto de alterações fisiológicas do envelhecimento, denominado de "senescência", sabendo diferenciá-las das alterações do envelhecimento patológico ou senilidade ${ }^{37}$, garantindo, assim, um bom acolhimento dessa população.

Idosos participantes da pesquisa qualitativa desenvolvida por Nery et al..$^{38}$ (2010) elencaram algumas dificuldades existentes no acesso à atenção odontológica: marcação de consultas, disponibilidade de vagas, tempo de espera, barreiras no atendimento às emergências, entre outros. No trabalho de Ramos e Lima ${ }^{39}$ (2003), foram apresentados alguns fatores que poderiam interferir no acesso aos serviços de saúde, dentre os quais a distância entre a unidade de saúde e o local de moradia do indivíduo, além do tempo e dos meios utilizados para o deslocamento.

Os aspectos citados anteriormente, em ambos os trabalhos, referem-se à acessibilidade. Travassos e Martins ${ }^{40}$ (2004) citam o trabalho de Penchansky e Thomas $(1981)^{1}$, os quais identificam várias dimensões que compõem o conceito de acesso, dentre elas a acessibilidade, caracterizada pela adequação entre a distribuição geográfica dos serviços e dos pacientes. Starfield $(2002)^{41}$ destaca acessibilidade como um dos elementos estruturais relevantes à atenção primária, a qual envolve a localização do estabelecimento próximo da população que atende, os horários e dias em que está aberto para atender, o grau de tolerância para consultas não agendadas e o quanto a população percebe a conveniência desses aspectos da acessibilidade. Segundo a mesma autora, a "acessibilidade" possibilita que as pessoas cheguem aos serviços.

Esses aspectos podem explicar os resultados encontrados por Martins et al. ${ }^{12}$ (2008), de acordo com os quais os idosos residentes na zona rural utilizaram menos os serviços odontológicos. Esse quadro tende a se agravar no caso dos idosos que necessitam de várias sessões para concluir seus tratamentos e que dependem de amigos ou parentes para transportá-los, conforme relatado no trabalho de Gregory et al. ${ }^{18}$ (2012).

Diante do exposto, percebe-se que o serviço público necessita de uma reformulação, direcionando ações específicas aos problemas da terceira idade ${ }^{7}$. Os resultados do SB Brasil 2010 mostram que o perfil epidemiológico da saúde bucal dessa população, em nível de Brasil, é crítico. O acúmulo de cárie e doença periodontal não tratada e, principalmente, o elevado edentulismo - que demanda por reabilitação protética - requerem maior interesse em modificar o quadro de desatenção para com a saúde bucal do idoso brasileiro ${ }^{19}$.

\footnotetext{
Penchansky DBA, Thomas JW. The concept of access - definition and relationship to consumer satisfaction. Med Care 1981; 19:127-40.
}

\section{Considerações finais}

Os resultados encontrados no presente estudo permitem concluir que os idosos não têm acesso regular aos serviços odontológicos. Os baixos níveis de escolaridade e a consequente limitação do conhecimento sobre a importância dos cuidados com a saúde, comumente atrelados às condições desfavoráveis de renda e moradia - esta última, muitas vezes, distante dos serviços de saúde -, constituem-se nas principais barreiras do acesso dessa população a esses serviços.

É preciso ampliar o conhecimento do idoso sobre saúde bucal, com vistas a conscientizar e motivar esse segmento com relação ao uso dos serviços odontológicos de forma preventiva e regular, desfazendo a crença de que a utilização dos serviços odontológicos somente é possível em caso de dor ou desconforto na cavidade bucal, prática que caracterizava a odontologia excludente realizada no passado. Além disso, cabe aos serviços de saúde garantir e viabilizar o acesso, ampliando a oferta de serviços, melhorando o acolhimento e favorecendo a acessibilidade dos idosos, na medida em que se trata de um grupo prioritário, possuidor de seu próprio Estatuto, o qual deve ser respeitado.

A Estratégia Saúde da Família pode atuar como um fator facilitador desse acesso, uma vez que permite a propagação, pela equipe, de informações sobre saúde no âmbito domiciliar. Pode promover, ainda, a busca ativa de idosos, o que possibilita identificar necessidades de tratamento e diagnosticar precocemente as doenças bucais, favorecendo a manutenção dos dentes naturais nessa população. Na perspectiva de suprir a demanda dos idosos, faz-se necessária, também, a ampliação da oferta de serviços de atenção secundária e de reabilitação protética, corroborando, assim, para o cumprimento dos princípios éticos do SUS - Universalidade, Integralidade e Equidade - e a consequente promoção da qualidade de vida dos idosos brasileiros.

\section{Abstract}

Demographic transition in Brazil has implied in a substantial change in the design of the age pyramid, resulting from the increased life expectancy, since health services should be prepared to absorb this new demand. Regarding oral health of the Brazilian elderly population, its condition, as well as in several countries, is precarious. Objective: this study aimed to identify, through a literature review, the factors interfering in the access of elderly people to oral health services. Materials and method: the research was conducted on BVS, LILACS, SCiELO, and BBO databases using the keywords: access to health services; oral health; elderly. The inclusion criteria for the selection of articles included the presence of the full text in databases, publications that presented 
the term elderly as subject threshold, and articles published between 2001 and 2012. Results: the access of the elderly to oral health services does not occur in a regular manner. Low educational level, low income, edentulism, low supply of public dental services, lack of information about oral health, and residing in rural areas may have a negative impact on the access of the elderly to oral health services. Final considerations: limitations related to the socioeconomics aspects represent the main barrier to the access of the elderly to oral health services.

Keywords: Health services access. Dental care for the elderly. Health inequalities. Elderly. Oral health.

\section{Referências}

1. Ferreira ABH. Miniaurélio: o minicicionário da língua portuguesa. 6. ed. rev. e atualiz. Curitiba: Positivo; 2004.

2. Jesus WLA, Assis MMA. Revisão sistemática sobre o conceito de acesso nos serviços de saúde: contribuições do planejamento. Ciênc Saúde Coletiva 2010; 15(1):161-70.

3. Brasil. Diretrizes e recomendações para o cuidado integral de doenças crônicas não transmissíveis: promoção da saúde, vigilância, prevenção e assistência. Brasília: Ministério da Saúde; 2008.

4. Instituto Brasileiro de Geografia e Estatística (IBGE). População brasileira deve chegar ao máximo (228,4 milhões) em 2042 [citado 2013 Ago 30]. Disponível em URL: http://saladeimprensa.ibge.gov.br/noticias?view=noticia\&id=1\&bus$\mathrm{ca}=1 \&$ idnoticia $=2455$.

5. Teixeira CF. Transição epidemiológica, modelo de atenção à saúde e previdência social no Brasil: problematizando tendências e opções políticas. Ciênc Saúde Coletiva 2004; 9(4):841-50.

6. Nespe-Fiocruz-UFMG. Carta de Ouro Preto: desigualdades sociais, de gênero e saúde dos idosos no Brasil. Belo Horizonte: UFMG; 2002 [citado 2012 Jul 05]. Disponível em URL: http://www.crde-unati.uerj.br/doc_gov/destaque/ ouro_preto.doc.

7. Colussi CF, Freitas SFT. Aspectos epidemiológicos de saúde do idoso no Brasil. Cad Saúde Pública 2002; 18(5):1313-20.

8. Gaião LR, Almeida MEL, Heukelback J. Perfil epidemiológico da cárie dentária. Rev Bras Epidemiol 2005; 8(3):316-23.

9. Nickel DA, Lima FG, Silva BB. Modelos assistenciais em saúde bucal no Brasil. Cad Saúde Pública 2008; 24(2):241-46.

10. Brasil. Projeto SBBrasil 2010: Pesquisa Nacional de Saúde Bucal: resultados principais. Brasília: Ministério da Saúde; 2011.

11. Manhães ALD, Costa AJL. Acesso a e utilização de serviços odontológicos no Estado do Rio de Janeiro, Brasil, em 1998: um estudo exploratório a partir da Pesquisa Nacional por Amostra de Domicílios. Cad Saúde Pública 2008 jan; 24(1):207-18.

12. Martins AMEBL, Barreto SM, Pordeus IA. Características associadas ao uso de serviços odontológicos entre idosos dentados e edentados no Sudeste do Brasil: Projeto SB Brasil. Cad Saúde Pública 2008; 24(1):81-92.
13. Rocha RACP, Goes PSA. Comparação do acesso aos serviços de saúde bucal em áreas cobertas e não cobertas pela Estratégia Saúde da Família em Campina Grande, Paraíba, Brasil. Cad Saúde Pública 2008; 24(12):2871-80.

14. Costa IMD, Maciel SML, Cavalcanti, AL. Acesso aos serviços odontológicos e motivos da procura por atendimento por pacientes idosos em Campina Grande-PB. Odontol Clín Científ 2008; 7(4):331-5.

15. Baldani MH, Brito WH, Lawder JAC, Mendes YBE, Silva FFM, Antunes JLF. Determinantes individuais da utilização de serviços odontológicos por adultos e idosos de baixa renda. Rev Bras Epidemiol 2010; 13(1):150-62.

16. Alcarde ACB, Bittar TO, Fornazari DH, Meneghim MC, Ambrosano GMB, Pereira AC. A cross-sectional study of oral health-related quality of life of Piracicaba's elderly population. Rev Odonto Ciênc 2010; 25(2):126-31.

17. Alcântara CM, Dias CA, Rodrigues SM, Reis FA. Estudo comparativo da condição de saúde bucal de idosos não institucionalizados de Governador Valadares-MG, com a meta proposta pela organização Mundial de saúde para 2010. PHYSIS: Rev Saúde Coletiva 2011; 21(3):1023-44.

18. Gregory J, Thomson WM, Broughton JR, Cullinan MP, Seymour GJ, Kieser JA, et al. Experiences and perceptions of oral health and oral health care among a sample of older New Zealanders. Gerodontology 2012; 29:54-63.

19. Moreira RS, Nico LS, Tomita NE, Ruiz T. A saúde bucal do idoso brasileiro: revisão sistemática sobre o quadro epidemiológico e acesso aos serviços de saúde bucal. Cad Saúde Pública 2005; 21(6):1665-75.

20. Wamala S, Merlo J, Boström G. Inequity in access to dental care services explains current socioeconomic disparities in oral health: The Swedish National Surveys of Public Health 2004-2005. J Epidemiol Community Health 2006; 60:1027-33.

21. Martins AMEBL, Barreto SM, Pordeus IA. Uso de serviços odontológicos entre idosos brasileiros. Rev Panam Salud Publica $2007 ; 22(5): 308-16$

22. Brasil. Portaria no 2.528 de 19 de outubro de 2006. Aprova a Política Nacional de Saúde da Pessoa Idosa. Brasília: Ministério da Saúde; 2006.

23. Fadel CB, Schneider L, Moimaz SAS, Saliba NA. Administração pública: o pacto pela saúde como uma nova estratégia de racionalização das ações e serviços em saúde no Brasil. Rev Adm Pública 2009; 43(2):445-56.

24. Ministério da Saúde. Portaria $n^{\circ}$ 399. Divulga o Pacto pela Saúde 2006 - Consolidação do SUS e aprova as Diretrizes Operacionais do Referido Pacto. Diário Oficial da União, Brasília, 22 fev 2006, seção 1. p. 43-51.

25. Ayres J, Calazans GJ, Saletti Filho HC, França Jr I. Risco, vulnerabilidade e práticas de prevenção e promoção da saúde. In: Campos G, Minayo MCS, Akerman M, Drumond Jr M, Carvalho YM. Tratado de saúde coletiva. São Paulo: Fiocruz; 2006. p. 375-417.

26. Instituto Brasileiro de Geografia e Estatística - IBGE. Síntese de Indicadores Sociais. Uma análise das condições de vida da população brasileira 2010. Rio de Janeiro: Ministério do Planejamento, Orçamento e Gestão; 2010.

27. Machado LP, Camargo MBJ, Jeronymo JCM, Bastos GAN. Uso regular de serviços odontológicos entre adultos e idosos em região vulnerável no sul do Brasil. Rev Saúde Pública $2012 ; 46(3): 526-33$ 
28. Viana AAF, Gomes MJ, Carvalho RB, Oliveira ERA. Acessibilidade dos idosos brasileiros aos serviços odontológicos. RFO UPF 2010; 15(3):319-24.

29. Neri M, Soares W. Desigualdade social e saúde no Brasil. Cad Saúde Pública 2002; 18 Suppl:77-87.

30. Instituto Brasileiro de Geografia e Estatística - IBGE. Pesquisa Nacional por Amostra de Domicílios. Um Panorama da Saúde no Brasil. Acesso e utilização dos serviços, condições de saúde e fatores de risco e proteção à saúde 2008 [citado 2013 Ago 31]. Disponível em URL: http://biblioteca. ibge.gov.br/visualizacao/monografias/GEBIS\%20\%20RJ/panorama.pdf.

31. Chaimowicz F. A saúde dos idosos brasileiros às vésperas do século XXI: problemas, projeções e alternativas. Rev Saúde Pública 1997; 31(2):184-200.

32. Rodrigues NO, Neri AL. Vulnerabilidade social, individual e programática em idosos da comunidade: dados do estudo FIBRA, Campinas, SP, Brasil. Ciênc Saúde Coletiva 2012; 17(8):2129-39.

33. Matos DL, Giatt, L, Lima Costa MF. Fatores sócio-demográficos associados ao uso de serviços odontológicos entre idosos brasileiros: um estudo baseado na Pesquisa Nacional por amostras de domicílio. Cad Saúde Pública 2004; 20(5):1290-7.

34. Brasil. Envelhecimento e saúde da pessoa idosa. Brasília: Ministério da Saúde; 2006.

35. Santos DLC, Superti L, Macedo MS. Acolhimento: qualidade de vida em saúde pública. Boletim da Saúde 2002; 16(2):30-51.

36. Brasil. Estatuto do idoso: lei federal $\mathrm{n}^{\mathrm{o}} 10.741$, de $01 \mathrm{de}$ outubro de 2003. Brasília: Secretaria Especial dos Direitos Humanos; 2004.

37. Ministério da Saúde. Plano de cuidado para idosos na saúde suplementar. [citado 2013 Ago 31]. Disponível em: http:// bvsms.saude.gov.br/bvs/publicacoes/plano_cuidado_idosos. pdf.

38. Nery NG, Marcelo VC, Dantas MAA. A satisfação de idosos quanto à estratégia saúde da família, a partir da atenção em saúde bucal. R Bras Ci Saúde 2010; 14(1):43-50.

39. Ramos DD, Lima MADS. Acesso e acolhimento aos usuários em uma unidade de saúde de Porto Alegre, Rio Grande do Sul, Brasil. Cad Saúde Pública 2003; 19(1):27-34.

40. Travassos C, Martins M. Uma revisão sobre os conceitos de acesso e utilização de serviços de saúde. Cad Saúde Pública 2004; 20 Suppl 2:S190-98.

41. Starfield B. Uma estrutura para a medição da Atenção Primária. In: Starfield B. Atenção Primária: equilíbrio entre necessidades de saúde, serviços e tecnologia. Brasília: Organização das Nações Unidas para a Educação, a Ciência e a Cultura/Ministério da Saúde; 2002. p. 43-71.

\section{Endereço para correspondência:}

Sílvia Helena de Carvalho Sales-Peres

Departamento de Odontopediatria, Ortodontia e Saúde Coletiva.

Faculdade de Odontologia de Bauru, Universidade de São Paulo (USP)

Al. Dr. Octávio Pinheiro Brisola, 9-75

17012-901 Bauru - SP

Fone: (14) 3235-8256

E-mail: shcperes@usp.br

Recebido: 15/07/2013. Aceito: 18/12/2013. 